\title{
Model and design of dielectric elastomer minimum energy structures
}

\author{
Samuel Rosset, Oluwaseun A Araromi, Jun Shintake and Herbert R Shea \\ École Polytechnique Fédérale de Lausanne (EPFL) Maladière 71b, 2002 Neuchâtel, Switzerland \\ E-mail: samuel.rosset@a3.epfl.ch
}

Received 7 January 2014, revised 15 May 2014

Accepted for publication 16 May 2014

Published 2 July 2014

\begin{abstract}
Fixing a prestretched dielectric elastomer actuator (DEA) on a flexible frame allows transformation of the intrinsic in-plane area expansion of DEAs into complex three-dimensional (3D) structures whose shape is determined by a configuration that minimizes the elastic energy of the actuator and the bending energy of the frame. These stuctures can then unfold upon the application of a voltage. This article presents an analytical modelling of the dielectric elastomer minimal energy structure in the case of a simple rectangular geometry and studies the influence of the main design parameters on the actuator's behaviour. The initial shape of DEMES, as well as the actuation range, depends on the elastic strain energy stored in the elastomeric membrane. This energy depends on two independent parameters: the volume of the membrane and its initial deformation. There exist therefore different combinations of membrane volume and prestretch, which lead to the same initial shape, such as a highly prestretched thin membrane, or a slightly prestretched thick membrane. Although they have the same initial shape, these different membrane states lead to different behaviour once the actuation voltage is applied. Our model allows one to predict which choice of parameters leads to the largest actuation range, while specifying the impact of the different membrane conditions on the spring constant of the device. We also explore the effects of non-ideal material behaviour, such as stress relaxation, on device performance.
\end{abstract}

Keywords: soft actuator, model, electrostatic, minimum energy structure

(Some figures may appear in colour only in the online journal)

\section{Introduction}

Dielectric elastomer actuators (DEAs) are a class of soft actuators capable of large strain (more than $100 \%$ area strain have been reported $[1,2])$. They are stretchable capacitors formed by a soft and thin (typically 20-50 $\mu \mathrm{m}$ ) elastomeric membrane sandwiched between two compliant electrodes. Upon application of a voltage on the electrodes, the electrostatic attraction causes a squeezing of the membrane, which decreases in thickness and increases in area. Both actuation mechanisms can be used directly. Thus, the area expansion can be used to move or deform an object placed on the membrane [3, 4], and the thickness compression can be used to make contractile stacked actuators, whose length contracts when activated [5,6]. More complex behaviours can be obtained with out-of-plane motion caused by membrane buckling [7], unimorph structures [8], through the use of local stiffeners [9], or with dielectric elastomer minimum energy structures (DEMES) [10-12], which were first introduced by Kofod et al.

DEMES consist of a flexible (but not stretchable) frame on which a prestretched DEA is attached. When external constraints are removed, part of the elastic energy stored into the prestretched membrane is transfered into the frame, which bends out of plane, taking a 3D equilibrium configuration characterized by the minimum energy of the system (bending of frame and stretching the membrane). The device is designed so the active area (i.e. with electrodes) of the elastomer membrane coincides with the hole(s) in the frame, i.e. where the membrane is suspended. The application of a voltage between the electrodes of the actuator changes the stress state of the membrane, and thus its strain energy, which causes the whole structure to unfold towards a new minimum energy configuration. Figure 1 presents the underlying 


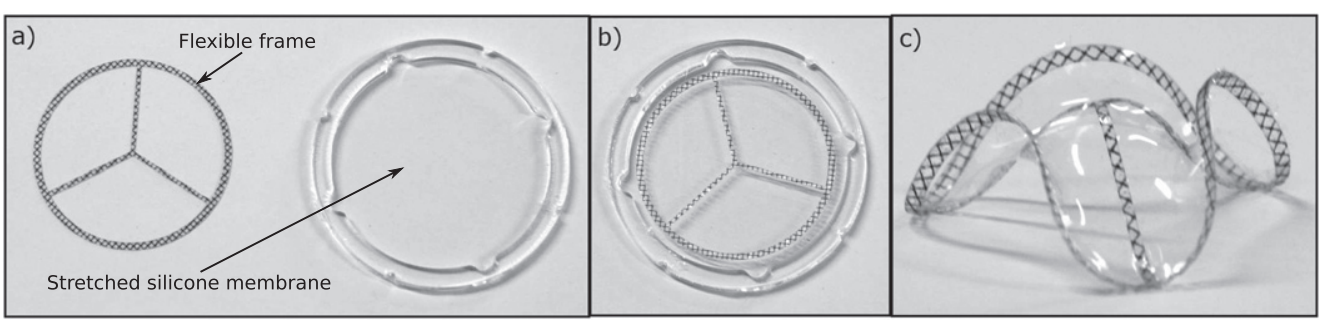

Figure 1. Complex shapes obtained by minimum energy structures. (a) a flexible (but not stretchable) frame is cut in a plastic sheet, and a membrane is prestretched and fixed on a holder to retain the tension. (b) the frame is glued to the prestretched membrane. (c) After separating the membrane from the holder, the frame + membrane assembly takes a complex 3D shape defined by the minimum energy configuration of the two components.

principle behind DEMES, starting from a frame and a prestretched membrane (a), which are glued together (b), and finally removed from the part holding the membrane. This causes relaxation of the device into a complex configuration (c). The figure shows a membrane without electrodes, so the structure presented in figure 1(c) cannot be actuated. To make a DEMES, it is necessary to apply compliant electrodes on both sides of the membrane, either at stage (a) or (b), when the membrane is still flat. There could be a single electrode pair covering the complete membrane, in which case the whole structure unfolds upon application of a voltage, or three electrically independent electrode pairs can be patterned in each of the three sectors, which allows each of the legs to unfold independently. Our fabrication process to prestretch and attach elastomeric membranes on flexible frames and to pattern compliant electrodes has been detailed in previous publications [13-15]. In this work, we consider a membrane prestretched in a pure shear condition (its width is kept constant when stretched along its length). We have built a stretcher capable of stretching membranes in this particular state, as described in [15].

This principle of voltage-controlled unfolding of flexible structures has been proposed for different applications, such as grippers $[13,16]$ or arrays of cilia acting as a conveyor [17]. Recently our lab has been investigating the possibility of using soft DEMES-based actuators as a gripping system for CleanSpace One, a project from the EPFL Space Center consisting in using a small-sized satellite equipped with a gripper and a propulsion system to bring space debris back into the atmosphere [14, 18]. For these applications with precise requirements, such as force and stroke, it is crucial to understand the impact of the main design parameters (frame stiffness, membrane material, thickness and prestretch, as well as the overall shape and size) on the performance of a device in order to design the most efficient actuator. Because of the complexity of the shape taken by the frame/membrane assembly, an analytical model is difficult to derive for an arbitrary actuator shape, and a finite element approach has been the preferred method $[17,19]$. However, because of the geometry of a DEMES, which consists of a membrane of a few square centimetres in surface but only a few tens of micrometers of thickness, a large number of elements are necessary, which leads to time-consuming simulations. To circumvent this issue, O'Brien et al have used membrane elements to model the dielectric elastomer membrane, thus drastically reducing the number of required nodes, and hence the computing time [19]. However, such elements are designed to model a state of in-plane stress and can therefore not directly include the impact of the vertical Maxwell pressure generated by the application of a voltage. O'Brien et al have written their own in-plane strain energy function, which includes the effect of the Maxwell pressure [19]. Even if this approach is much faster than using solid elements (the computation time and gain are not given), it remains ill-adapted to explore a wide, multi-dimensional parameter space.

Even though finite element analysis is the most convenient method to obtain quantified results for a complex DEMES geometries, simpler geometries can be studied analytically. For example, a rectangular frame with a rectangular hole at its center can be used to make a gripper, when two actuators are mounted facing each other (figure 2). Kofod et al presented an analytical model to describe the behaviour of a DEMES shell actuator consisting of an elastomeric membrane stretched across a flexible frame, which buckles into a portion of a cylinder when relaxing the the minimum energy point [16]. However, the model was not used to optimize the actuators, nor to study the impact of the main parameters on the behaviour of the devices.

In this contribution, we use a minimum-energy approach to analytically calculate the equilibrium position of rectangular DEMES under different conditions. The theoretical model allows us to study the qualitative impact of the different design parameters on the behaviour of the structure. In particular, given a frame with a defined size and material, what should be the properties of the elastomeric membrane in order to maximize the actuation stroke and the blocking force. For example, the elastic strain energy stored in the membrane can be increased with a higher prestretch, or a higher thickness, thus meaning that there are several combinations of prestretch and membrane thickness leading to the same equilibrium angle. If we take two different DEMES with the same initial rest angle when no voltage is applied, one with a thick membrane but slightly prestretched, and one with a thinner membrane but highly prestretched, how will they behave when actuated? Which one exhibits the highest stroke or the highest blocking force? This is what our study aims to answer. We also investigate the impact of non-ideal behaviour 


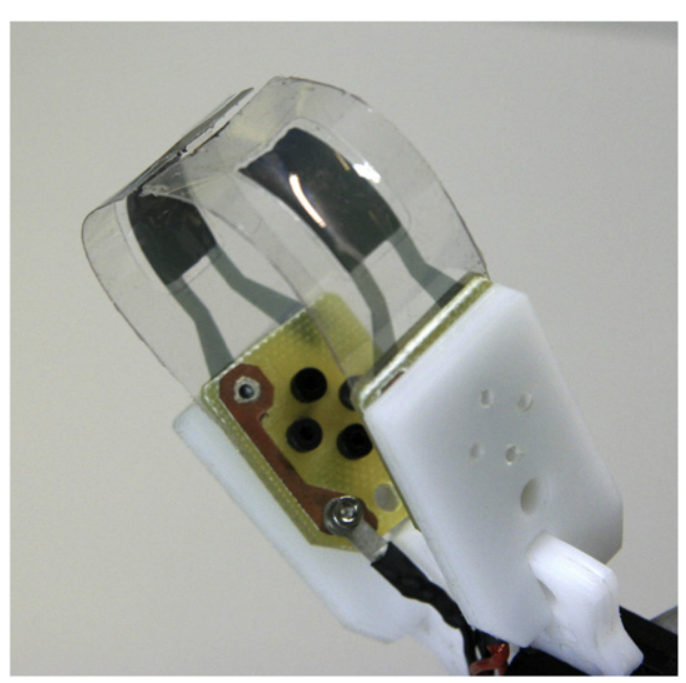

Figure 2. Example of a two-finger gripper made with DEMES. In this case the frame geometry simply consists of a rectangle with a rectangular hole at the center.

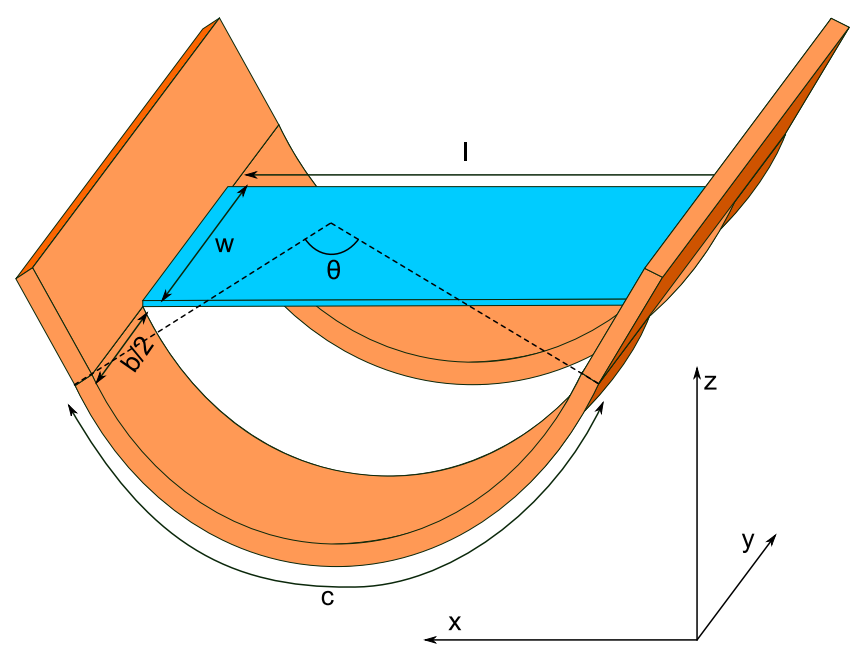

Figure 3. DEMES geometrical configuration: a frame with a rectangular hole of dimension $c \times w$ and two bending arms, each of them of size $b / 2 \times c$. The membrane is stretched across the hole and has a size $l \times w$. The curved length of the frame is linked to the membrane length $l$ by the bending angle of the frame $\theta$.

of the materials, such as plastic creep in the frame, and stress relaxation in the dielectric membrane.

\section{Analytical model}

The DEMES (figure 3) is modelled by a rectangular frame with a rectangular hole at its center. The important dimensions and material parameters are defined in table 1 . They are the width of the hole $w$ (which is also the width of the membrane), the length of the hole $c$ (which deforms into a sector of a circle of angle $\theta$ ), the width of the bending portion of the frame, two stripes of $b / 2$ each, and the thickness of the frame $d$. In addition, the frame is also characterized by the Young's modulus of the material it is made of $\left(Y_{f}\right)$. The
Table 1. Definition of the different variables used to describe the geometry and mechanical properties of the frame and the membrane.

\begin{tabular}{|c|c|}
\hline$c$ & length of the bending part of the frame \\
\hline$b$ & $\begin{array}{l}\text { width of the bending part of the frame (separated in two } \\
\text { arms of width } b / 2 \text { ) }\end{array}$ \\
\hline$d$ & thickness of the frame \\
\hline$Y_{f}$ & Young Modulus of the frame \\
\hline$\theta$ & bending angle of the frame \\
\hline$w$ & width of the membrane \\
\hline$l, l_{0}$ & stretched and initial length of the membrane \\
\hline$t, t_{0}$ & stretched and initial thickness of the membrane \\
\hline$\mu, J_{m}$ & Gent model mechanical parameters for the membrane \\
\hline
\end{tabular}

membrane has the same width $w$ as the hole in the frame, and a length which depends on the bending angle of the frame $\theta$ as given by (1), with the particular case $l(0)=c$.

$$
l=\frac{2 \cdot c}{\theta} \sin \frac{\theta}{2}
$$

For this simplified model, the membrane is bridging the two extremities of the bending portion of the frame, whereas in reality, the membrane, being also fixed along the $c$ boundary of the frame, takes a more complex saddle shape. However, this simplification is necessary in order to have a simple membrane shape whose strain energy can be calculated. We expect the impact of this approximation to be negligible for a first approach, especially at smaller angles $\theta$ and for wide membranes $(w>c)$.

To calculate the bending energy $U_{b}$ of the frame as a function of angle, we assume a constant bending moment along the frame:

$$
U_{b}=\frac{1}{2} \frac{Y_{f} \cdot b \cdot d^{3}}{12 \cdot c} \theta^{2}=\frac{1}{2} k_{b} \cdot \theta^{2},
$$

with $k_{b}$ the bending spring constant, by analogy with a linear spring. The stiffness of the frame can therefore be tuned by modifying its geometry, or by changing the material it is made of.

\subsection{Membrane strain energy}

Elastic energy is stored in the membrane by prestretching it before fixing it to the frame. In order to bend the frame along the $y$ axis (see figure 3), pure shear prestretch is used (figure 4). Thus, the width of the membrane is kept constant and equal to the hole width $w$. The initial length of the membrane $l_{0}$ is stretched until the membrane reaches the size of the hole in the frame $(w \times c)$, at which point it is fixed to the frame. Under these conditions, and taking the membrane incompressibility into account $\left(\lambda_{x} \lambda_{y} \lambda_{z}=1\right)$, the prestretches in the membrane, when it is applied on the flat frame, are given by:

$$
\lambda_{p x}=\lambda_{p}=\frac{c}{l_{0}}, \lambda_{p y}=1, \lambda_{p z}=\frac{1}{\lambda_{p}},
$$

where the indices $p$ and $x, y, z$ refer respectively to prestretch 


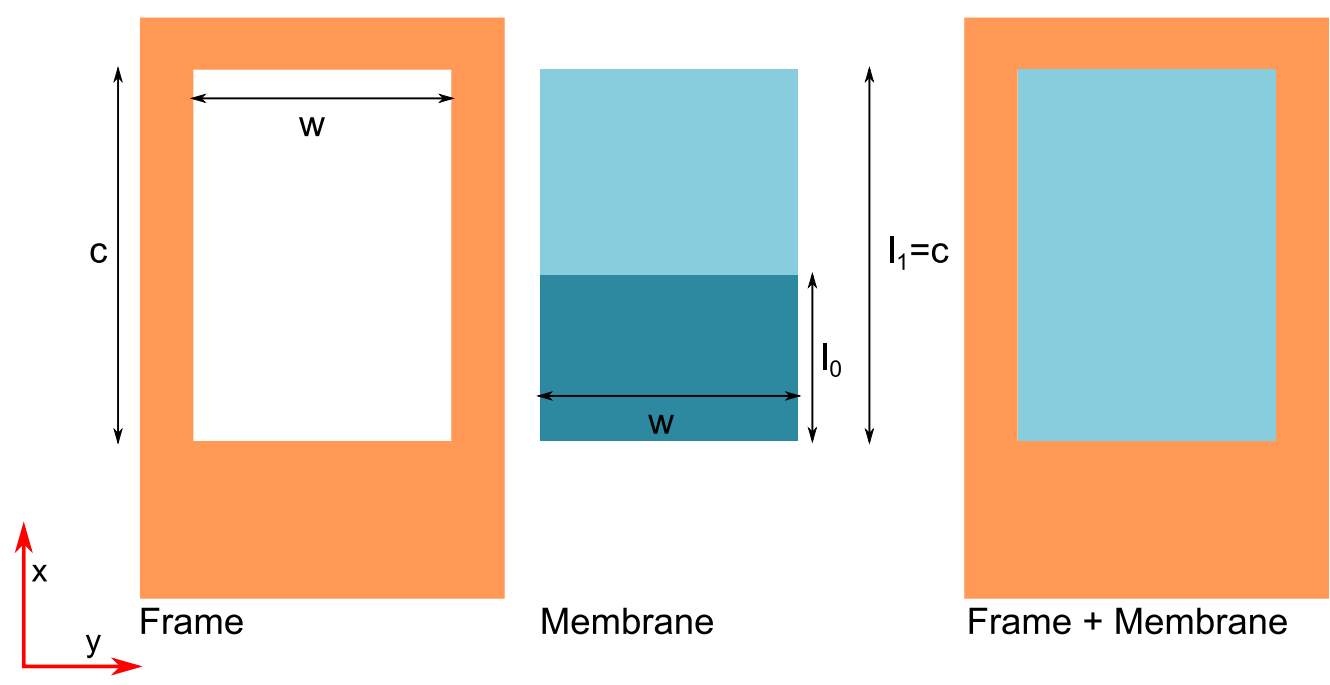

Figure 4. Prestretching the membrane in pure shear: the membrane with an initial length $l_{0}$ and an initial width $w$ is stretched to a final length $l_{1}$ which corresponds to the length of the frame hole $c$. The width of the membrane is kept at the initial value $w$.

and the principal directions as defined in figure 3. Consequently, the initial thickness of the membrane $t_{0}$ is reduced upon prestretching to $t_{1}=t_{0} / \lambda_{p}=t_{0} l_{0} / c$. The advantage of using a pure shear prestretching condition over other particular cases such as equi-biaxial or uniaxial prestretch comes from the fact that when the frame is relaxed from its initial flat state and bends to its equilibrium position, or is electrically activated, its stretching condition remains in pure shear, thus making the modelling easier. In addition, unlike equi-biaxial prestrain, it promotes bending predominantly along the $y$ axis, and the parasitic bending along $x$ caused by the non-zero stress about the $y$ direction can easily be suppressed by strengthening the extremities of the frame. Uniaxial prestretching along $x$ only causes bending about the $y$ axis, but when the frame bends and the membrane relaxes, this generates compressive stress in the membrane in the $y$ direction, which leads to undesired buckling of the membrane.

The prestretching of the membrane described in figure 4 is only a schematic representation of the situation. In reality, for the fabrication of DEMES, the membrane is not of the exact size of the hole, but larger than the entire frame, because the membrane must be attached to the frame and must therefore cover it in order to provide a common surface for adhesion. In our fabrication process [15], we use a dedicated stretching rig that can prestretch a large membrane (larger than the frame) in pure shear. The frame is then adhered to the stretched membrane. The latter is then cut around the external perimeter of the frame to release the device and allow it to bend out of plate and take its rest position. If the frequently used acrylic elastomer VHB from $3 \mathrm{M}$ is used as dielectric membrane, then adhesion between the frame and the membrane is provided by the adhesive nature of the membrane $[10,12]$. In the case of silicone membranes, an adhesive must be applied on the frame before laying it on the prestretched membrane. However, its impact on the frame stiffness and bending energy (2) must be taken into account. To avoid this issue, we have also used oxygen plasma treatment to bond silicone membranes to both PET and metallic foils. The adhesion strength is not as good as when using $\mathrm{O}_{2}$ plasma to bond silicone on itself, glass or silicon, but sufficient to sustain the prestretch and prevent delamination from the frames.

The stretch state given by (3) is a particular case obtained when the frame is flat. When it bends, the general stretch state in the three directions becomes:

$$
\lambda_{x}=\lambda=\frac{l}{l_{0}}, \lambda_{y}=1, \lambda_{z}=\frac{1}{\lambda} .
$$

Combining together equations (1) and (4) leads to:

$$
\lambda(\theta)=\frac{2 c}{\theta l_{0}} \sin \frac{\theta}{2} .
$$

Out of the different hyperelastic material models that can be used to describe the stretch-stress behaviour of elastomers, the Gent model [20] is particularly interesting, as it predicts the divergence of the stress to an infinite value for a finite stretch value, thus representing the maximal finite elongation an elastomer can reach before breaking due to the finite length of its polymer chains. Consequently, it is often used in DEA modelling $[2,21,22]$. The strain energy density $u$ according to the Gent model is given by:

$$
u=\frac{-\mu J_{m}}{2} \ln \left(1-\frac{\lambda_{x}^{2}+\lambda_{y}^{2}+\lambda_{z}^{2}-3}{J_{m}}\right),
$$

with $\mu$ the shear modulus of the material, $J_{m}$ is a material constant describing the limiting stretch. For the particular case of pure shear (4), the strain energy density can be rewritten as:

$$
u=\frac{-\mu J_{m}}{2} \ln \left(1-\frac{\lambda^{2}+\lambda^{-2}-2}{J_{m}}\right) .
$$

The elastic strain energy $U_{e l}$ is obtained by multiplying the energy density (7) with the volume of the membrane. Because of their very high bulk modulus, the volume of elastomer 
remains constant when deformed, and it is therefore only necessary to calculate the volume in one particular configuration, for example, before the prestretching step, when the membrane is in its reference state:

$$
\begin{aligned}
U_{e l}= & l_{0} \cdot w \cdot t_{0} \cdot u=-\frac{c \cdot w \cdot t_{0}}{\lambda_{p}} \frac{\mu J_{m}}{2} \\
& \times \ln \left(1-\frac{\lambda^{2}+\lambda^{-2}-2}{J_{m}}\right) .
\end{aligned}
$$

To take the effect of the electric-field induced Maxell pressure on the membrane strain energy into account, we assume that the elastomer behaves as an ideal dielectric so that the total energy in the membrane is the sum of the elastic energy and the electrostatic energy [2]. The electrostatic energy $U_{e s}$ is defined as:

$$
U_{e s}=-\frac{C \cdot V^{2}}{2}=-\frac{\epsilon \cdot c \cdot w \cdot V^{2} \lambda^{2}}{2 t_{0} \lambda_{p}},
$$

where $C$ is the capacitance of the device, $V$ is the applied voltage, and $\epsilon$ is the permittivity of the dielectric. The minus signs comes from the fact that in constant voltage operation, the energy is provided by the external voltage source to the actuator.

Finally, the total energy of the system $U_{\text {tot }}$ is the sum of the frame-bending energy (2), and the membrane-free energy (8) and (9):

$$
\begin{aligned}
& U_{t o t}(\theta, V)=\frac{1}{2} \frac{Y_{f} \cdot b \cdot d^{3}}{12 \cdot c} \theta^{2}-\frac{c \cdot w \cdot t_{0}}{\lambda_{p}} \frac{\mu J_{m}}{2} \\
& \times \ln \left(1-\frac{\lambda^{2}+\lambda^{-2}-2}{J_{m}}\right)-\frac{\epsilon \cdot c \cdot w \cdot V^{2} \lambda^{2}}{2 t_{0} \lambda_{p}}
\end{aligned}
$$

using (5) to express the stretch $\lambda$ as a function of the bending angle $\theta$. The equilibrium angle is found by minimizing equation (10), which is equivalent to solving:

$$
\frac{\partial U_{t o t}}{\partial \theta}=0
$$

and controlling that the obtained solution corresponds to a local minimum.

\section{Application of the model}

\subsection{Frame and membrane}

We used the analytical model presented above by calculating the behaviour of a realistic DEMES. We chose a frame made out of $25 \mu \mathrm{m}$ steel with a Young modulus $Y_{f}=200 \mathrm{GPa}$. Steel was chosen for the numerical application because it behaves elastically and is less subject to creep than plastic thin foils (see section 3.3). However, it should be noted that the exact same bending stiffness would be obtained for a $110 \mu$ m-thick PET frame. The width of each beam $b / 2$ was equal to $3 \mathrm{~mm}$, and the hole cut into the steel shim was $w \times c=20 \times 10 \mathrm{~mm}^{2}$.
For the elastomeric membrane, and in order to obtain a relevant set of parameters, we conducted an experimental pull-test on a silicone membrane with Dow Corning Sylgard 186. Because the membrane of the considered structure was in a pure shear condition, a pure shear pull test was performed in order to obtain the two material parameters of the Gent model (6). This was done by using a sample much wider than its length, so as to avoid reduction of the sample width during the test and to ensure a pure shear condition [23]. Our samples were $110 \times 10 \mathrm{~mm}^{2}$, the smallest dimensions being placed in the pulling direction. The thickness of the samples was $38 \mu \mathrm{m}$. Figure 5 presents the results of the pure shear pull test together with a fit of the Gent hyperelastic model. The parameters of the model extracted from the curve fitting were $\mu=0.16 \mathrm{MPa}$ and $J_{m}=18.3$. Two pull tests were performed on different samples and led to the same model parameters. The fit was performed for stretch values between 1 and 3, which are of interest for the present study, even though it was possible to mechanically stretch the sample to higher values. However, at higher stretch values the Gent model starts to diverge from the data, and the parameter values that we give here for Sylgard 186 are therefore valid only for pure shear stretch $<3$. It can be seen on figure 5 that the Gent model fits the data very well in this stretch range.

As there are many parameters that influence the actuator behaviour, we decided to fix the frame parameters (geometry and material), as well as the membrane material (using the Gent model parameters presented above for Dow Corning Sylgard 186). The remaining degrees of freedom were therefore related to the geometry of the membrane. When the membrane is fixed on the frame (i.e. when $\theta=0$, which is equivalent to $\lambda=\lambda_{p}$ ), the elastic strain energy (8) can be increased by two different means. The first method occurs by increasing the prestretch, which the strain energy density, but decreases the volume of the membrane. However, as the strain density increases faster than the volume decreases, a larger prestretch does indeed contribute to an increase of the strain energy. The second method involves increasing the volume of the membrane for a given prestretch-induced stress also increases the stored elastic energy. This can be done either by increasing the width of the membrane or by increasing its thickness. It effectively increases the membrane cross-section, and therefore the elastic restoring force in the membrane. While these last two parameters have the same effect on the calculation of the elastic energy (8), they have different implications. For example, increasing the width increases the overall surface of the device, which can be undesirable depending on the application. Additionally, if $w$ becomes large, it then becomes more difficult to prevent the frame from bending about the $x$ axis. On the other hand, increasing the thickness has no influence on the volume occupied by the device. However, the voltage required to drive the device increases, but this can be addressed by using a multilayered active membrane [5]. An additional advantage of varying the membrane thickness as opposed to its width is the possibility of using the exact same frame to test different configurations. Consequently, we chose the membrane 


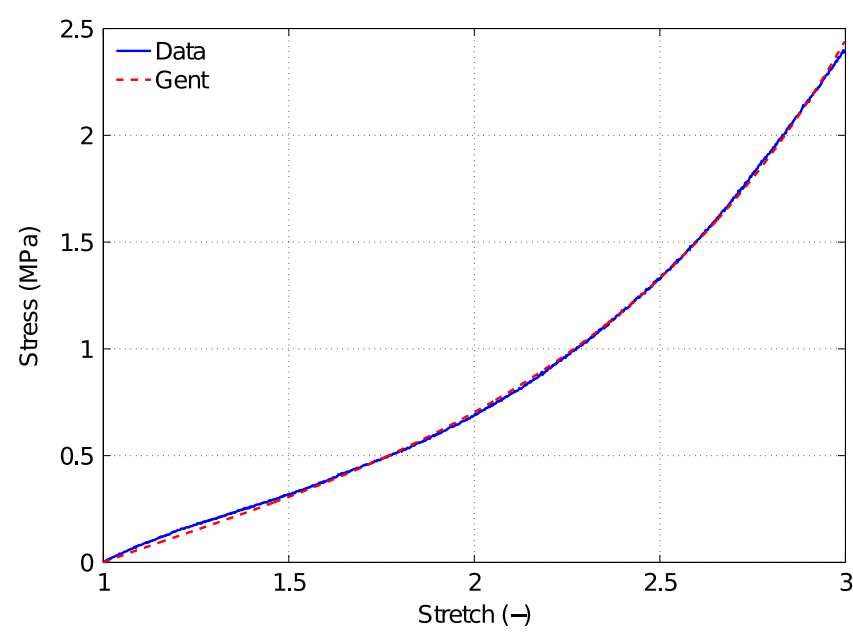

Figure 5. Pure shear pull test on a Dow Corning Sylgard 186 sample and fit with the Gent hyperelastic material model.

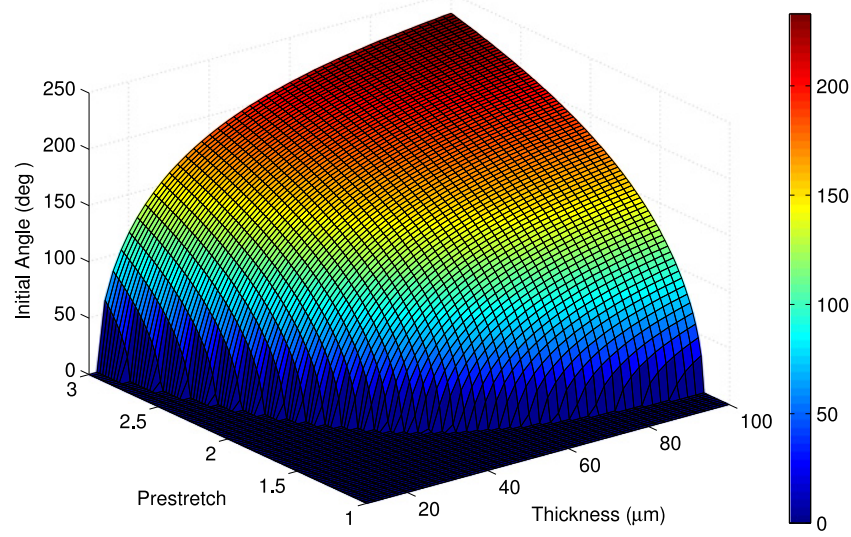

Figure 6. Initial bending angle in degrees for different membrane thickness and prestretch values.

prestretch $\lambda_{p}$ and the membrane thickness $t_{0}$ (which represents the thickness of the membrane as fabricated) as the two main design parameters whose influence on the performance of the device we want to investigate.

As discussed in detail in the next section, increasing the elastic energy (either by a larger prestretch or a thicker/wider membrane) increases the initial bending angle of the device because more energy is available in the membranes, which can be transferred to the frame.

\subsection{Initial angle and actuation range}

The first important parameter characterizing the DEMES is its rest angle or initial angle $\theta_{0}$, which represents how much the frame bends when the prestretched membrane is glued to the frame and the assembly is subsequently allowed to relax. The initial angle is obtained by solving equation (10) in the absence of electrostatic actuation $(V=0)$. The results (figure 6) show that a certain amount of strain energy is necessary for the frame to start bending, and for too low thickness or prestretch, the structure remains flat. $\mathrm{P}$ this threshold, an increase of strain energy (obtained either by increasing the prestretch or the thickness) leads to a larger initial bending angle, as expected. It can be noted that different pairs of prestrain and thickness values can lead to the same equilibrium angle. Given a targeted initial angle (e.g. $90^{\circ}$ ), one can either select a thin, highly prestretched membrane or a thick, slightly prestretched membrane, to take two examples at the extremes. However, the behaviour when activated differs, even though the rest angles are equal.

To evaluate the effect of actuation, we calculate the actuation range, i.e. the difference in bending angle between the rest position, and the position reached at the maximal voltage. The maximal voltage is the highest voltage that can be applied to the device without breakdown through the dielectric membrane. We assume that the elastomeric membrane of the actuator can be characterized by exhibiting a constant dielectric breakdown field $E_{b d}$ representing the highest electric field that the material can sustain without failure. We have measured breakdown fields around $100 \mathrm{~V} / \mu \mathrm{m}$ for thin Dow Corning Sylgard 186 membranes with little to no prestretch, similar to the conditions presented here [24]. In order to avoid destroying the device, we define a maximal field $E_{\max }$ with a given security margin relative to the breakdown field (typically $50 \%-80 \%$ of $E_{b d}$ ), representing the maximal field that can be applied to the device at any time. In our calculations, we choose a maximal field of $70 \mathrm{~V} / \mu \mathrm{m}$ in order to leave a safety margin with respect to the breakdown field. We therefore define the actuation range as the difference in bending angle between a situation where no field is applied across the elastomeric membrane $(E=0)$, and when the maximal field is applied to the device $\left(E=E_{\max }\right)$. However, the electric field applied to the device cannot be directly controlled by the user, and dielectric elastomer actuators are usually driven in a voltage-controlled mode. The electric field across the membrane is linked to the applied voltage and the current thickness of the membrane by:

$$
E=\frac{V}{t}=\frac{V \cdot \lambda}{t_{0}} .
$$

For a given applied voltage $V$ the electric field in the membrane is therefore at its maximal value when the membrane is flat, i.e. when $\lambda=\lambda_{p}$. Consequently, we define the maximal voltage $V_{\max }$ that can be applied to a device such as not to exceed $E_{\max }$ in the membrane at any time by:

$$
V_{\max }\left(t_{0}, \lambda_{p}\right)=\frac{E_{\max } \cdot t_{0}}{\lambda_{p}}
$$

As a consequence, for every point of the studied parameter space (see figure 6), each representing a different device, we have a different value of the maximal applied voltage, ranging from $233 \mathrm{~V}\left(10 \mu \mathrm{m}, \lambda_{p}=3\right)$ to $7000 \mathrm{~V}\left(100 \mu \mathrm{m}, \lambda_{p}=1\right)$. Because the electrostatic pressure $p$ on the device is proportional to the square of the electric field $\left.\left(p=\epsilon \cdot E^{2}\right)[1]\right)$, it means that we consider the same electrostatic force acting at each point in the parameter space, as they are each submitted to the same electric field when the maximal voltage is applied. 


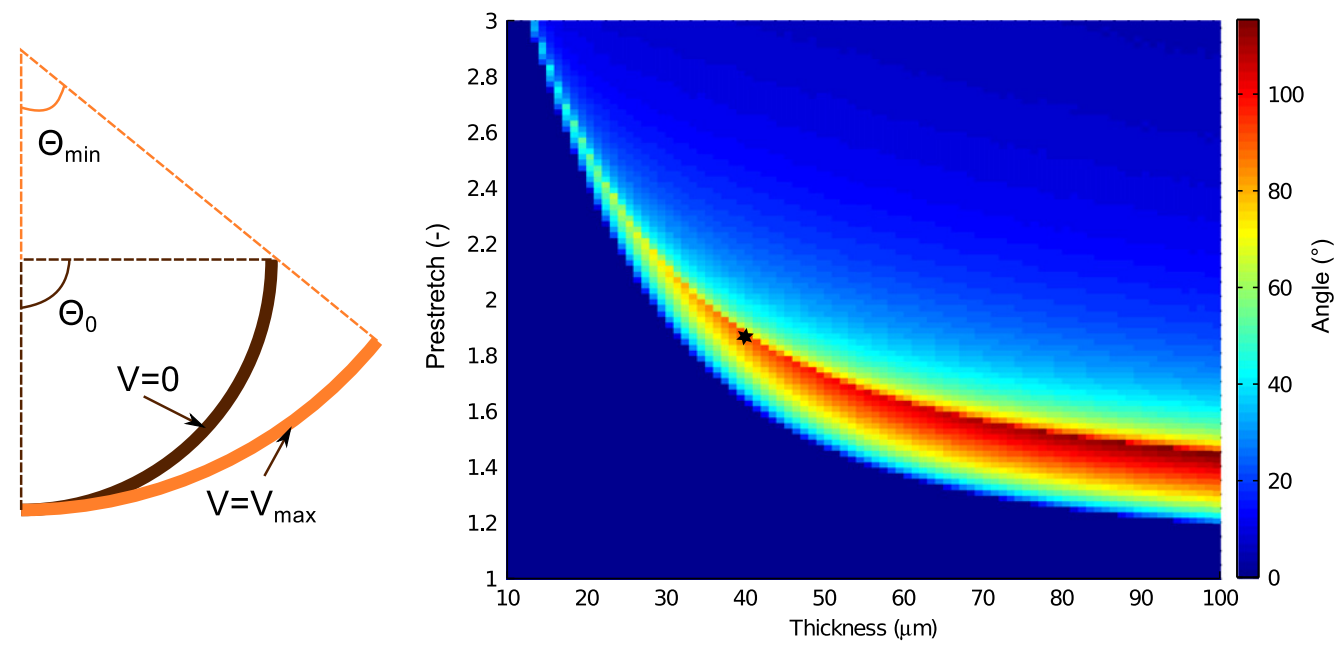

Figure 7. Maximal actuation range in degrees for different membrane thickness and prestretch values and an electric field of $70 \mathrm{~V} / \mu \mathrm{m}$. The actuation range is defined as the bending angle difference between the rest angle $\theta_{0}$ and the minimal angle $\theta_{\min }$ obtained at the maximal voltage $V_{\max }$. The black stars represents a device with a $40 \mu \mathrm{m}$ membrane at the optimal prestretch (for this thickness) of 1.86 .

The angle at the maximum voltage is calculated by solving (11) for $V=V_{\max }$ and the maximum change of angle a DEMES can provide is obtained by calculating the difference between the activated angle and the initial angle $\theta_{0}$ (figure 7). The results show the presence of a very sharp maximum ridge: for each thickness value, there is an optimal prestretch value $\lambda_{p, o p t}$ that maximizes the actuation angle. Prestretches even slightly different from the optimal value lead to a sharp drop in actuation performance. Furthermore, using thicker membranes with less prestretch is expected to lead to a larger change in angle.

For a $40 \mu \mathrm{m}$ membrane, the optimal prestretch is $\lambda_{p, o p t}=1.86$. For this particular case, the bending energy of the frame, the strain energy of the membrane, as well as the total energy of the system, are plotted on figure 8 . As seen on the figure, the maximal actuation angle is obtained when the actuator is designed to reach the flat position when the full voltage is applied. If the prestretch is lower, then the flat position is reached at a voltage smaller than $V_{\max }$ and a further increase of voltage has no effect on the actuation. Alternatively, if the prestretch is higher than the optimal value, the flat position cannot be reached at $V_{\max }$ and the actuation range is drastically reduced. This is caused by geometric effects: a small variation in the membrane length caused by the electrostatic actuation leads an important change of angle when the frame is flat. From (1), we see that $d \theta / d l$ is large when $\theta$ is close to 0 . In summary, for the chosen frame material and geometry, and for the chosen elastomer with a $40 \mu \mathrm{m}$ initial thickness, the optimal pure shear prestretch is 1.86 for maximum actuation range. The equilibrium angle is computed to be $90^{\circ}$ at $0 \mathrm{~V}$ and completely flat at the maximal field of $70 \mathrm{~V} / \mu \mathrm{m}$, which corresponds to an applied voltage of $1505 \mathrm{~V}$.

In addition to the actuation angle, another important parameter is the force that the DEMES can generate. For a gripper application, it is important for the actuator to be strong

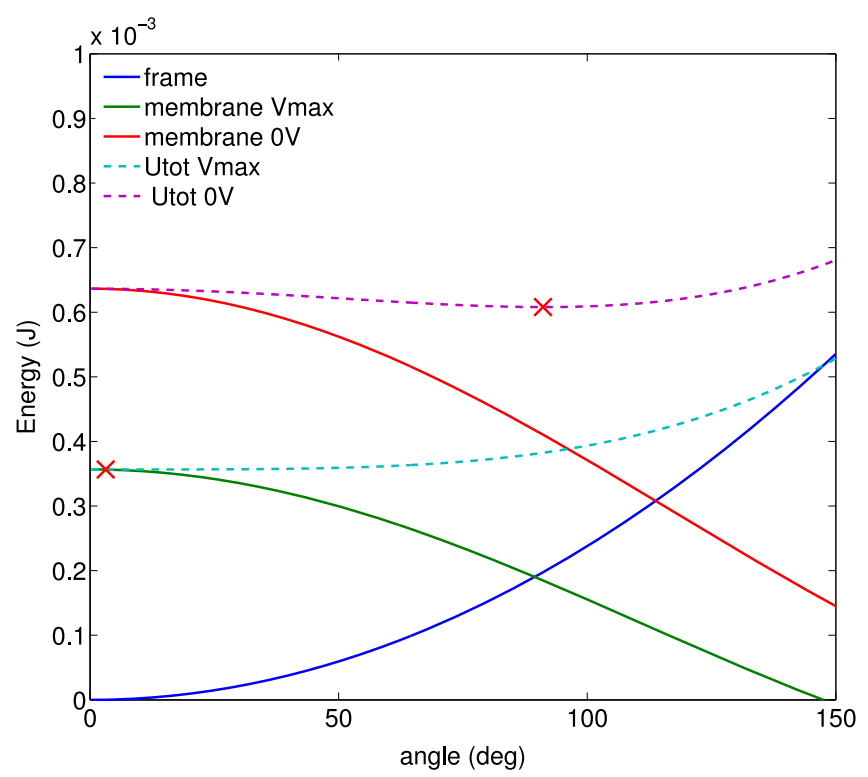

Figure 8. Bending energy of the frame, strain energy of the membrane and total energy of the system for $0 \mathrm{~V}$ and maximum voltage. The red crosses indicate the energy minima. $t_{0}=40 \mu \mathrm{m}$, $\lambda_{p}=1.86$.

enough to hold and manipulate objects. When the actuator is at an equilibrium position $\theta$, it acts as a spring whose voltagedependent spring constant can be calculated by differentiating the energy twice with respect to the angle. This is valid for small deformations perpendicular to the frame, around the equilibrium position. For the particular case of figure 8, the local spring constant is $0.15 \mathrm{mN} /$ degree when no voltage is applied and $0 \mathrm{mN} /$ degrees at $V=V_{\max }$. This later value is due to the flat energy landscape around the equilibrium position when the maximal voltage is applied. This is systematically observed when the actuator is designed to reach the flat position when fully activated, making them especially 


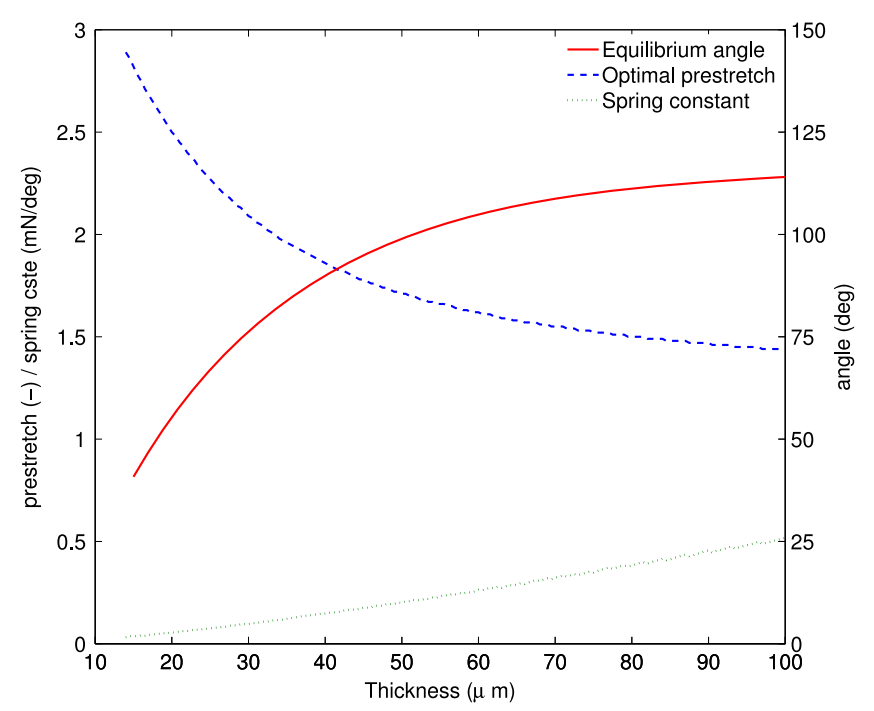

Figure 9. Optimal prestretch value, local spring constant at $0 \mathrm{~V}$ and rest angle for different membrane thickness.

sensitive to external perturbations, as will be discussed in more detail later (cf section 4).

The initial bending angle, maximal actuation angle change and spring constant at the rest position depend on the membrane thickness and prestrain. On figure 9, the optimal prestretch is given for a range of initial membrane thickness. The initial bending angle in these conditions (also equal to the actuation range, as the actuator can be unfolded up to $\theta=0$ ) and the local spring constant in the inactivated state are also shown. It can be seen that using a thicker membrane increases both the tuning range and the spring constant and should therefore be favoured. There are two factors limiting how thick the actuator should be. The first one is the actuation voltage (13) which increases with membrane thickness (this can be solved by using multilayered actuators as already mentioned). The second is that the stress within the membrane is smaller in the case of thicker membranes (the strain energy density is small, but it leads to a high strain energy because of the large volume of the membrane), and external perturbations could place the membrane in a compressive state. This would induce buckling of the membrane and cause a drastic reduction in spring constant.

\subsection{Non-ideal behaviour of the frame and the elastomer materials}

Because of the flat energy landscape around the flat position when the device is activated at $V_{\max }$, it is very sensitive to outside perturbation or variations in the fabrication. For example, if the prestretch is slightly off the calculated value, the actuation range will be much smaller than predicted. There are two main possible causes of discrepancy between the theoretical model presented above and real devices: first, stress relaxation in the dielectric membrane (viscoelastic creep) and second, permanent plastic deformation of the frame (creep).
3.3.1. Stress relaxation in the membrane. Because elastomers are viscoelastic materials, they have a ratedependent stress-stretch relationship and are subject to stress relaxation. For example, when a membrane is prestretched and fixed on a frame, stress relaxation takes place, which causes a time-dependent decrease of the elastic energy stored in the stretched membrane. In a DEMES configuration this would cause the equilibrium angle of the structure to decrease over time. A stress relaxation characterization of the widely used acrylic elastomer VHB from $3 \mathrm{M}$ has been performed by Wissler and Mazza [25]. Because of the viscoelastic nature of VHB, the stress in the sample still noticeably decreases after $1000 \mathrm{~s}$. Michel et al performed similar tests on VHB and a silicone elastomer (Dow Corning 3481) and showed that due to much smaller viscoelastic losses, the silicone elastomer reaches a stable stress state much faster than VHB [26]. To confirm the results for the silicone we are using in this study (Dow Corning Sylgard 186), we have performed a stress relaxation test in the exact same pure shear conditions that were used for the characterization of the hyperelastic material behaviour (cf section 3.1). The sample was stretched in pure shear up to a stretch of $\lambda=2.5$ and $\lambda=3$ in 1 second, and held in this position (figure 11). One can see that the stress within the sample quickly reached a steady-state value, and that the value of the peak a $t=0$ is only about $6 \%$ higher than the steady-state value, thanks to the low-loss factor of silicones. For DEMES applications using silicone as a dielectric membrane, stress relaxation in the membrane is expected to have a negligible impact on device performance.

3.3.2. Creep in the frame. One of the major causes of discrepancy between the theoretical model presented above and real devices is the fact that the frame is considered to be a perfect spring. However, in reality the frame is subjected to plastic creep when it is kept in a bent position. This is especially true of plastic frames, which are often used for DEMES, because they can be easily cut to the desired geometry (see e.g. figure 1 or $[10,11,16,17])$. We previously used polyimide and PET frames to make DEMES [13, 14], and noticed an increase of the initial angle of our DEMES over a period of several weeks. As stress relaxation (cf 3.3.1) causes a decrease of the equilibrium angle, the observed degradation can be attributed to the frame. To confirm this, we conducted some accelerating ageing tests on PET frames, without bonded elastomeric membranes: frames were rolled around a post and placed in an oven for the night at $80^{\circ}$. Once detached from the post, the frames exhibited a significant residual bending angle (figure 10), proving the existence of creep behaviour in the material.

We include creep in the model by defining a creep angle $\theta_{c}$ representing the angle at which the bending energy of the frame is null. For an ideal frame not subjected to creep, $\theta_{c}=0$, which corresponds to the results shown in figures 6-9. As an illustration, we computed the maximal actuation angle in the case of a frame with a residual angle of $20^{\circ}$ (figure 12). These results are to be compared with figure 7 , which 


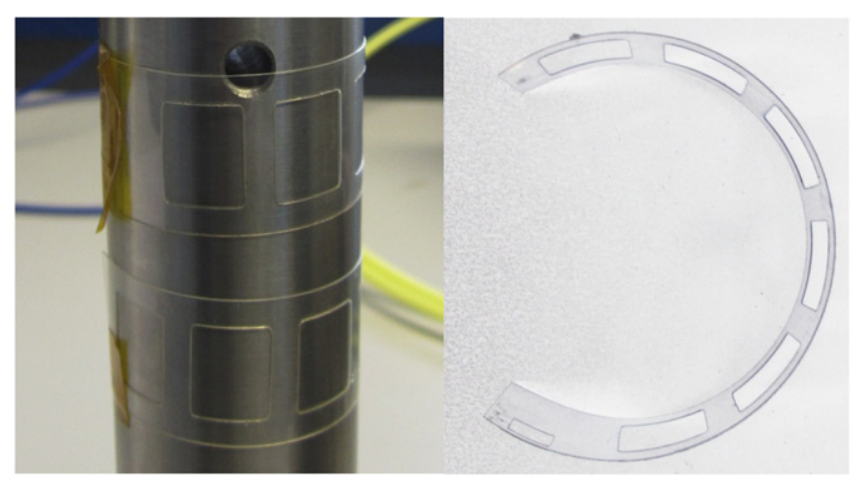

Figure 10. PET frames rolled around a post and left for one night at $80^{\circ}$ (left) exhibited a significant creep when released from the post (right).

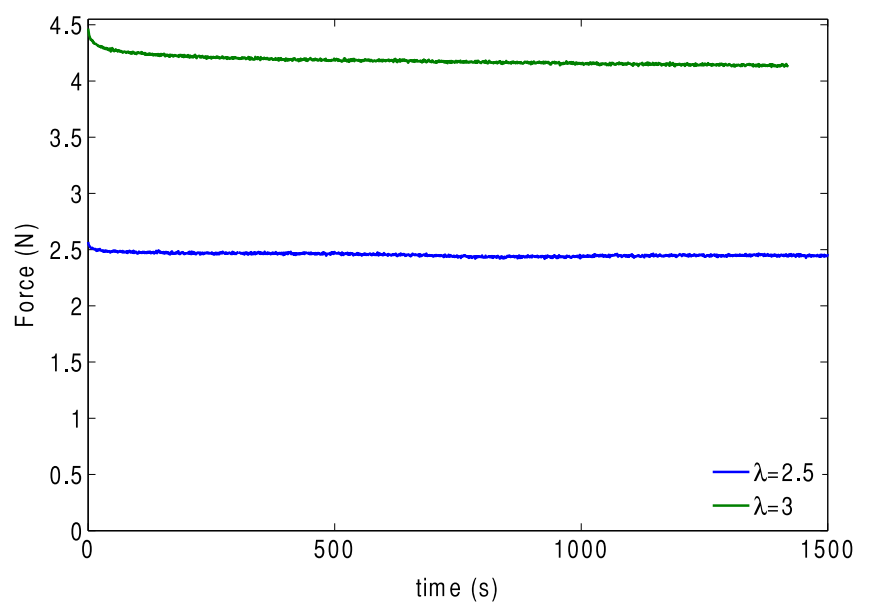

Figure 11. Stress relaxation test for the silicone Dow Corning Sylgard 186. The $110 \times 10 \mathrm{~mm}^{2}$ sample is stretched in pure shear to two different stretch values: $\lambda=2.5$ and $\lambda=3$ in 1 second, and held in this position. A relatively small stress relaxation of about $6 \%$ is observed, but a steady-state value is achieved after a few tens of seconds.

corresponds to the same situation, but without creep. It can be seen that creep drastically reduces the maximum tuning range for the optimal prestretch at each thickness by roughly a factor of two. We also found that the optimum ridge is much broader than for the case without creep, meaning that the maximum actuation angle becomes less sensitive to fabrication errors and imprecisions (membrane thickness and prestretch). The broadening of the peak is a geometric effect; creep prevents to work around the flat position, a zone in which a small membrane strain leads to an important change in the frame's bending angle. Furthermore, the optimal prestretch for each thickness value is slightly reduced. Additionally, by analogy with the ideal case, for which in the largest tuning range is obtained when the actuator becomes completely flat at the maximum voltage, one would expect to see the actuation angle being maximized when the final angle reaches $\theta_{c}$. However, this is not the case (figure 13). Taking the same $40 \mu \mathrm{m}$ membrane as before (cf section 3.2 ), the optimal prestretch is now 1.66. The energy landscape predicts an initial bending angle at $0 \mathrm{~V}$ of $93^{\circ}$ and a maximal

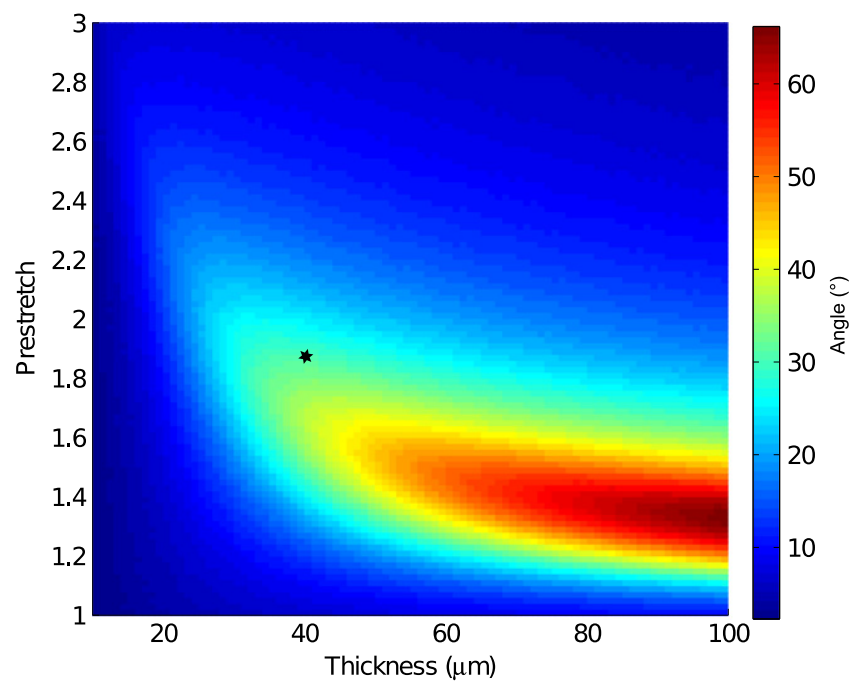

Figure 12. Actuation angle (difference between bending angle at $0 \mathrm{~V}$ and at $V_{\max }$ ) for a creep angle $\theta_{c}=20^{\circ}$. The black star represents the same device than on figure 7 ( $40 \mu \mathrm{m}$, prestretch of 1.86), which is an optimal combination in the absence of creep. When creep is taken into account, the chosen thickness/prestretch combination does not correspond to the optimum anymore.

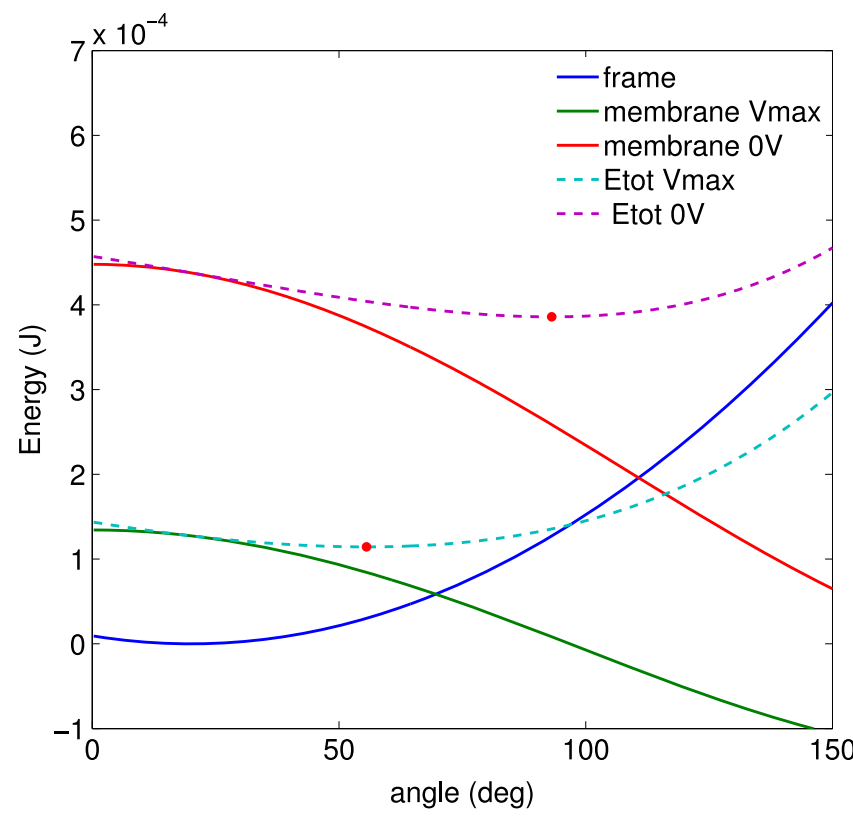

Figure 13. Energy landscape for a creep angle of $\theta_{c}=20^{\circ}$ for a $40 \mu \mathrm{m}$ membrane at the optimal prestretch $\left(\lambda_{p}=1.66\right)$. The red dots indicate the energy minima.

actuation range of $38^{\circ}$ for an activated angle of $55^{\circ}$, thus larger than $\theta_{c}$. However, unlike the perfect frame situation, the total energy at the maximum voltage is not flat around the equilibrium point, leading to a non-zero spring constant and an actuator much less sensitive to external perturbation in its activated state. A comparison of the parameters obtained for the ideal and creep cases is given in table 2 .

One of the most important impacts of creep on the behaviour of a DEMES actuator is that its performance can change with time. For example, a DEMES can be designed 
Table 2. Comparison of different parameters for a $40 \mu \mathrm{m}$ membrane at optimal prestretch for the case of no creep in the frame and $20^{\circ}$ creep in the frame

\begin{tabular}{lcc}
\hline & no creep & $\theta_{c}=20^{\circ}$ \\
\hline Optimal $\lambda_{p}$ & 1.86 & 1.66 \\
Initial angle $\left(^{\circ}\right)$ & 90 & 93 \\
Final angle $\left(^{\circ}\right)$ & 0 & 55 \\
Actuation range $\left(^{\circ}\right)$ & 90 & 38 \\
Spring cst. 0 V & 0.15 & 0.2 \\
Spring cst. $V_{\max }$ & 0 & 0.14 \\
\hline
\end{tabular}

for optimal performance (i.e. on the black star on figure 7), and therefore display a large actuation range just after fabrication. However, because of the creep occurring in the frame over time, the maximal actuation range landscape will be modified, and the actuator (black star on figure 12) won't be located on the maximal performance zone anymore, and consequently, its tuning range will be noticeably reduced.

This effect is demonstrated in figure 14 with a DEMES made with a polyimide frame. The superimposed pictures of the actuator both after fabrication and forty-six days later shows the increase of the initial angle without applied voltage. The graphs show the bending angle as a function of the applied voltage. Just after fabrication, the actuation angle range was $59.8^{\circ}$, while forty-six days later, it was reduced to $33.9^{\circ}$.

\section{Discussion}

In the absence of creep in the frame, the largest tuning range in DEMES is obtained if it is designed to reach the flat position at the maximal admissible voltage, and by an adequate selection of prestrain, given the membrane material, thickness, and frame size. However, in this particular position, the spring constant of the device is null. The importance of this property depends on the application. For a gripper, such as the one being developed to grab a small satellite [14], it is important that the DEMES structures present a high enough spring constant when the actuators are not activated (i.e. in the closed configuration), as they must be strong enough to prevent the captured object from escaping. When the arms of the gripper are open, their spring constant is of secondary importance. However, it means that the unfolded DEMES is very sensitive to external perturbations, which can unexpectedly modify the equilibrium position, as shown by the very flat energy landscape around the equilibrium position in figure 8. If this situation is undesired, then the actuator should be designed to reach $\theta>0$ (i.e. not flat) for the maximal voltage, thus ensuring a positive spring constant at any equilibrium position, but at the cost of a reduced tuning range. This situation is likely to occur on real devices for two different reasons. First, as explained above (cf 3.3), creep induced in the flexible frame shifts the the minimum of the bending energy in the frame to $\theta>0$. But as creep is a mechanism that depends on many factors (frame material, temperature, time, etc.), it is hard to control and should be minimized by a careful selection of the frame material. Second, unlike the simplified situation used for this model, in which the elastomer membranes are only present in the hole of the frame (cf figure 4), real devices have the membrane covering the complete frame, on which adhesive is applied to hold the membrane in place. This creates a unimorph structure in the bending arms of the frame, which are indeed a bilayer formed by the frame material and have the prestretched membrane bonded to it. This structure has an equilibrium position for an angle $\theta>0$, leading to a behaviour similar to what was presented in the case of creep.

DEMES can be used as soft grippers, as demonstrated by Kofod et al [16]. In this case, both a large actuation range and large initial equilibrium angle are desirable. However, as this study shows, a high initial equilibrium bending angle leads to a smaller actuation range. In addition, large initial angles pose the practical problem of the dielectric membrane occupying most of the useful volume of the gripper. For the satellite gripping application mentioned in the introduction and requiring long and narrow 'fingers,' we solved both issues by segmenting the actuator. Instead of having a single hole in the frame, several smaller holes were cut along the frame [14]. This is equivalent to mechanically placing several DEMES in series, the total initial angle being the sum of the initial angle of each sub-unit. With this approach, a large equilibrium angle $>180^{\circ}$ can be obtained, combined with a large actuation range, and without having the membrane taking up too much useful space.

\section{Conclusions}

We show that a simplified analytical model of a dielectric elastomer minimum energy structure allows rapid prediction of the behaviour of such actuators given the different geometrical and mechanical parameters of the device, as well as applied voltage. For a frame with defined dimensions, we have investigated the influence of the strain energy stored in the elastomeric membrane, which can be modulated by the applied voltage. We show that for each membrane thickness, there is an optimal prestretch value that allows one to maximize the tuning range of the device, and that the use of thicker membranes with lower prestretch leads to a larger tuning range, especially in the case of non-perfect frames whose rest position is not flat.

In order to obtain a set of equations easy to implement and solve, a number of simplifications have been made in the modelling. In addition, practical applications of a DEMESbased actuator will probably take advantage of the complex 3D shapes that can be obtained with this concept, and use a geometry more complex than a simple rectangle (see, for instance, the gripper developed by Kofod et al [16]). Although the present model cannot be directly applied to such complex geometries, whose modelling requires the use of FEM, the general qualitative trends reported in the manuscript also apply, and show that the parameters of the elastomeric 

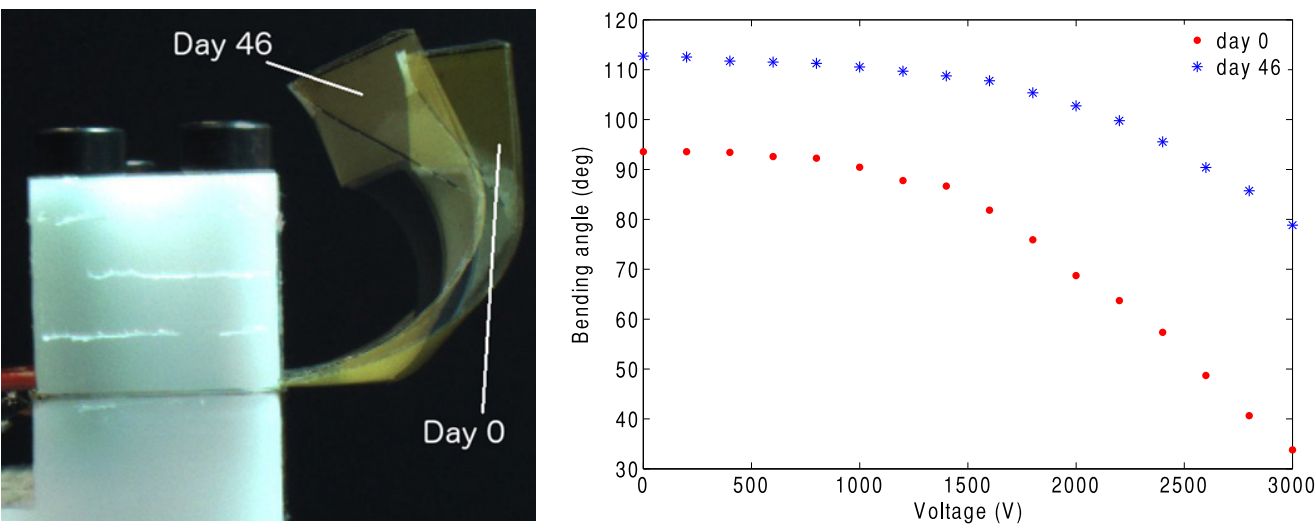

Figure 14. Left: Superimposed picture of a DEMES in its resting position (no voltage applied) as fabricated (day 0), and forty-six days later. Creep in the frame causes the initial angle to increase. Right: Actuation angle versus applied voltage for the actuator directly after fabrication and forty-six days later. The tuning range is much smaller after forty-six days because of the creep in the frame material.

membrane stretched on a frame (thickness and prestretch) must be carefully chosen in order to reach the desired actuation range and spring constant. This study thus provides a design guide for DEMES allowing the optimization of such structures for a given task.

\section{Acknowledgments}

This project was funded by the Swiss National Science Foundation, grant number 200020-140394 and 200020153122.

\section{References}

[1] Pelrine R, Kornbluh R, Pei Q and Joseph J 2000 High-speed electrically actuated elastomers with strain greater than 100\% Science 287 836-9

[2] Keplinger C, Li T, Baumgartner R, Suo Z and Bauer S 2012 Harnessing snap-through instability in soft dielectrics to achieve giant voltage-triggered deformation Soft Matter $\mathbf{8}$ 285-8

[3] Anderson I A, Hale T, Gisby T, Inamura T, McKay T, O'Brien B, Walbran S and Calius E P 2010 A thin membrane artificial muscle rotary motor Appl. Phys. A 98 75-83

[4] Kollosche M, Doering S, Stumpe J and Kofod G 2011 Voltagecontrolled compression for period tuning of optical surface relief gratings Opt. Lett. 36 1389-91

[5] Kovacs G and During L 2009 Contractive tension force stack actuator based on soft dielectric eap Proc. SPIE $\mathbf{7 2 8 7}$ 72870A

[6] Carpi F, Salaris C and Rossi D D 2007 Folded dielectric elastomer actuators Smart Mater. Struct. 16 300-5

[7] Rosset S, Niklaus M, Dubois P and Shea H R 2009 Largestroke dielectric elastomer actuators with ion-implanted electrodes J. Microelectromech. Syst. 18 1300-8

[8] Araromi O A, Conn A T, Ling C S, Rossiter J M, Vaidyanathan R and Burgess S C 2011 Spray deposited multilayered dielectric elastomer actuators Sensors Actuators A 167 459-67

[9] Lai W, Bastawros A F and Hong W 2012 Out-of-plane motion of a planar dielectric elastomer actuator with distributed stiffeners Proc. SPIE 8340834011
[10] Kofod G, Paajanen M and Bauer S 2006 Self-organized minimum-energy structures for dielectric elastomer actuators Appl. Phys. A 85 141-3

[11] O'Brien B, Calius E, Xie S and Anderson I 2008 An experimentally validated model of a dielectric elastomer bending actuator Proc. SPIE 6927 69270T

[12] Petralia M T and Wood R J 2010 Fabrication and analysis of dielectric-elastomer minimum-energy structures for highlydeformable soft robotic systems 2010 IEEE/RSJ Int. Conf. on Intelligent Robots and Systems pp 2357-63

[13] Shintake J, Rosset S, Floreano D and Shea H 2013 Effect of mechanical parameters on dielectric elastomer minimum energy structures Proc. SPIE 868786872 V

[14] Araromi O A, Gavrilovich I, Shintake J, Rosset S, Richard M, Gass V and Shea H R Roll-able multisegment dielectric elastomer minimum energy structures for a deployable microsatellite gripper IEEE/ASME Trans. Mechatron. at press

[15] Araromi O A, Gavrilovich I, Shintake J, Rosset S and Shea H R 2014 Towards a deployable satellite gripper based on multisegment dielectric elastomer minimum energy structures Proc. SPIE $905690562 \mathrm{G}$

[16] Kofod G, Wirges W, Paajanen M and Bauer S 2007 Energy minimization for self-organized structure formation and actuation Appl. Phys. Lett. 90081916

[17] O'Brien B, Gisby T, Calius E, Xie S and Anderson I 2009 FEA of dielectric elastomer minimum energy structures as a tool for biomimetic design Proc. SPIE 7287728706

[18] Mohammadreza M 2012 Cleaning up Earth's orbit: A Swiss satellite tackles space debris Press Release http://actu.epfl. ch/news/cleaning-up-earth-s-orbit-a-swiss-satellite-tack-2/

[19] O'Brien B, McKay T, Calius E, Xie S and Anderson I 2009 Finite element modelling of dielectric elastomer minimum energy structures Appl. Phys. A 94 507-14

[20] Gent A N 1996 A new constitutive relation for rubber Rubber Chem. Technol. 69 59-61

[21] Akbari S, Rosset S and Shea H R 2013 Improved electromechanical behavior in castable dielectric elastomer actuators Appl. Phys. Lett. 102071906

[22] Li W and Landis C M 2012 Deformation and instabilities in dielectric elastomer composites Smart Mater. Struct. 21 094006

[23] Kollosche M, Zhu J, Suo Z and Kofod G 2012 Complex interplay of nonlinear processes in dielectric elastomers Phys. Rev. E 85051801

[24] Rosset S, Niklaus M, Stojanov V, Felber A, Dubois P and Shea H R 2008 Ion-implanted compliant and patternable 
electrodes for miniaturized dielectric elastomer actuators Proc. SPIE 6927 69270W

[25] Wissler M and Mazza E 2007 Mechanical behavior of an acrylic elastomer used in dielectric elastomer actuators Sensors Actuators A 134 494-504
[26] Michel S, Zhang X Q, Wissler C, Loewe M and Kovacs G 2010 A comparison between silicone and acrylic elastomers as dielectric materials in electroactive polymer actuators Polymer Int. 59 391-9 